\title{
Fluid-driven mechanical responses of deformable porous media during two-phase flows: Hele-Shaw experiments and hydro- mechanically coupled pore network modeling
}

\author{
Min-Kyung Jeon ${ }^{1}$, Amin Hosseini Zadeh ${ }^{2}$, Seunghee $\mathrm{Kim}^{2}$, and Tae-Hyuk Kwon ${ }^{*}$ \\ ${ }^{1}$ Korea Advanced Institute of Science and Technology, Daejeon, Republic of Korea \\ ${ }^{2}$ University of Nebraska-Lincoln, Omaha, NE, USA
}

\begin{abstract}
Injecting fluid into a porous material can cause deformation of the pore structure. This hydromechanically coupled (i.e., poromechanical) phenomenon plays an essential role in many geological and biological operations across a wide range of scales, from geologic carbon storage, enhanced oil recovery and hydraulic fracturing to the transport of fluids through living cells and tissues, and to fuel cells. In this study, we conducted an experimental and numerical investigation of the hydro-mechanical coupling during fluid flows in porous media at the fundamental pore-scale. First, experimental demonstrations were undertaken to ascertain the effect of the hydro-mechanical coupling for two-phase fluid flows in either deformable or non-deformable porous media. Next, a hydro-mechanically coupled pore network model (HM-PNM) was employed to test a various range of influential parameters. The HM-PNM results were consistent with the experimental observations, including the advancing patterns of fluids and the development of the poroelastic deformation, when the viscous drop was incorporated. The hydromechanical coupling was observed to reduce the inlet pressure required to maintain a constant flow rate, whereas its effect on the pattern of fluid flow was minimal. The interfacial tension alteration also changed the pressure and deformation. The viscosity of invading fluid showed significant effects on both the patterns of fluid displacement and mechanical deformation.
\end{abstract}

\section{Introduction}

The deformation of pore structures during fluid injection (or withdrawal) occurs in many energy- or environmentally relevant operations, such as hydraulic fracturing for enhanced oil/gas recovery, geothermal energy extraction, and geologic storage of carbon dioxide [1-4]. The theory of poroelasticity has been widely used to explain the concurrence of fluid flow and deformation of hosting porous media in a conceptually neat way. However, our understanding at the pore-scale level is still in its infancy [5-7].

Moreover, the injected fluids are generally immiscible with indigenous fluid in porous media. In these two-phase flow cases, many parameters should be considered, and it is difficult to consider on a continuum scale. The capillary number $(\mathrm{Ca})$ and viscosity ratio $(\mathrm{M})$ have been widely used to determine the fluid pattern in the two-phase flow [8-12]. However, most researches have been performed on two-phase flows in a stiff medium, where the interaction between fluid flow and the hosting porous media can be ignored. When the injection pressure is comparable or larger than medium stiffness, the fluiddriven deformation takes place. The deformation affects fluid flows simultaneously, but their interplay still remains poorly established.

In this study, we investigated the two-phase flows in deformable porous media via both experimental and numerical methods. In the laboratory, mineral-oil injection tests were conducted into water-saturated media packed with either hydrogel beads or glass beads to examine the effect of medium stiffness during two-phase flow. Single-phase fluid injection tests were also conducted using the same set of porous media for comparison using the mixture of water and glycerol. In the numerical simulation, the two-phase flows in both deformable and non-deformable media were then simulated by using the hydro-mechanically coupled pore network model (HM-PNM). In addition, the parametric study was implemented to discover the effects of fluid characteristics such as interfacial tension (IFT) and viscosity of injection fluid on flow patterns and the development of medium deformation.

\footnotetext{
* Corresponding author: t.kwon@kaist.ac.kr
} 


\section{Experimental Investigation}

\subsection{Materials and experimental setup}

In this study, glass beads (diameter $\sim 1.59 \mathrm{~mm}$ ) and hydrogel beads (Snow real polymer; average diameter $1.6 \mathrm{~mm}$ ) were used to construct the non-deformable and deformable porous medium of a single layer in a visualization cell. The porous medium was saturated with colored water $(2 \mathrm{~g} / \mathrm{L})$ for 24 hours before beginning the fluid injection test. Mineral oil (Fisher BioReagents ${ }^{\mathrm{TM}}$, viscosity $\sim 28 \mathrm{cp}$ ) was used as the injecting fluid to impose the two-phase fluid flow condition. Two separate test implementations were conducted with the deformable (hydrogels) and non-deformable (glass beads) porous medium to examine the influence of poromechanical deformation during the fluid flow in porous media. Single-phase fluid flow condition was also investigated as a reference case, where the mixture of water and glycerol was used as both the saturating and injecting fluid. The high viscosity of the water-glycerol mixture ( 13cp) enables the deformation of hosting solids during the fluid flow. Note that the experimental and the following numerical study here emulated the fluid flow in an uncemented porous medium that consists of individual solid grains.

The test setup includes a visualization cell, a syringe pump (Harvard PHD|ULTRA), a pressure transducer (Omega), a data acquisition system (Keysight), a digital camera (Canon EOS 80D), BD syringes, and plastic tubes as shown in Fig.1. The visualization cell was made of two circular transparent glass plates with a diameter of 280 $\mathrm{mm}$ and a thickness of $180 \mathrm{~mm}$ separated by a perforated rubber $(1.59 \mathrm{~mm}$ thickness). The perforated rubber confined the grains inside and provided a working area of $210 \mathrm{~mm}$ diameter inside the visualization cell. The only mechanical load exerted on the system is the self-weight of the upper visualization cell $(\sim 20 \mathrm{~N})$. The radial deformation is restrained by the perforated spacer.

The injection port (1 mm ID) connected to the BD plastic syringe was located in the bottom center of the visualization cell. The syringe pump injected the invading fluid into the cell through the injection port. A plastic Tconnection connects the pressure transducer to the injecting tube. All tests were conducted in the laboratory temperature $\left(21 \pm 1^{\circ} \mathrm{C}\right)$.

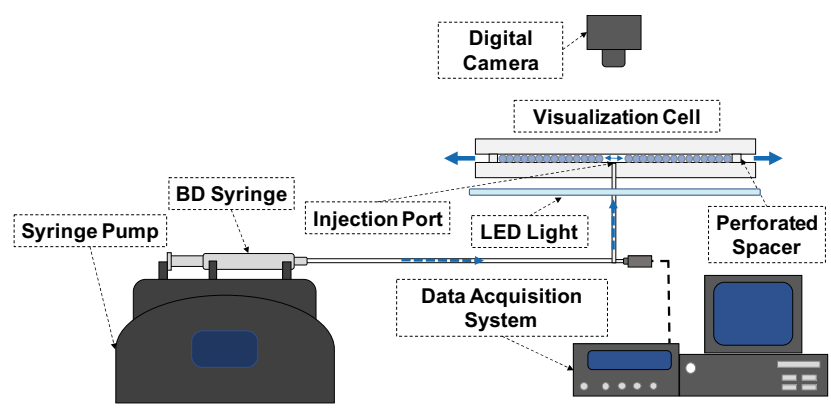

Fig. 1. A schematic illustration of the experimental setup.

\subsection{Test procedure}

In this experiment, the immiscible fluid with a higher viscosity (or same fluid) was injected into both deformable and non-deformable porous media. A monolayer of saturated glass/hydrogel grains was prepared inside the visualization cell between two glass plates, confined by the rubber spacer. Then a fluid was injected from the central injection port under a constant flow rate of $10 \mathrm{ml} / \mathrm{min}$ until the injected fluid percolated through the boundary. The pressure imposed at the inlet during the test was recorded using a pressure transducer connected to the injection port, and a digital camera located at the top of the cell captured the fluid flow pattern and the current deformation of solid grains in the porous medium.

\subsection{Results}

The fluid flow pattern and the deformation of solid grains are captured in Fig. 2a and Fig. 2b for the deformable and non-deformable porous media, respectively (immiscible two-phase fluid flow).
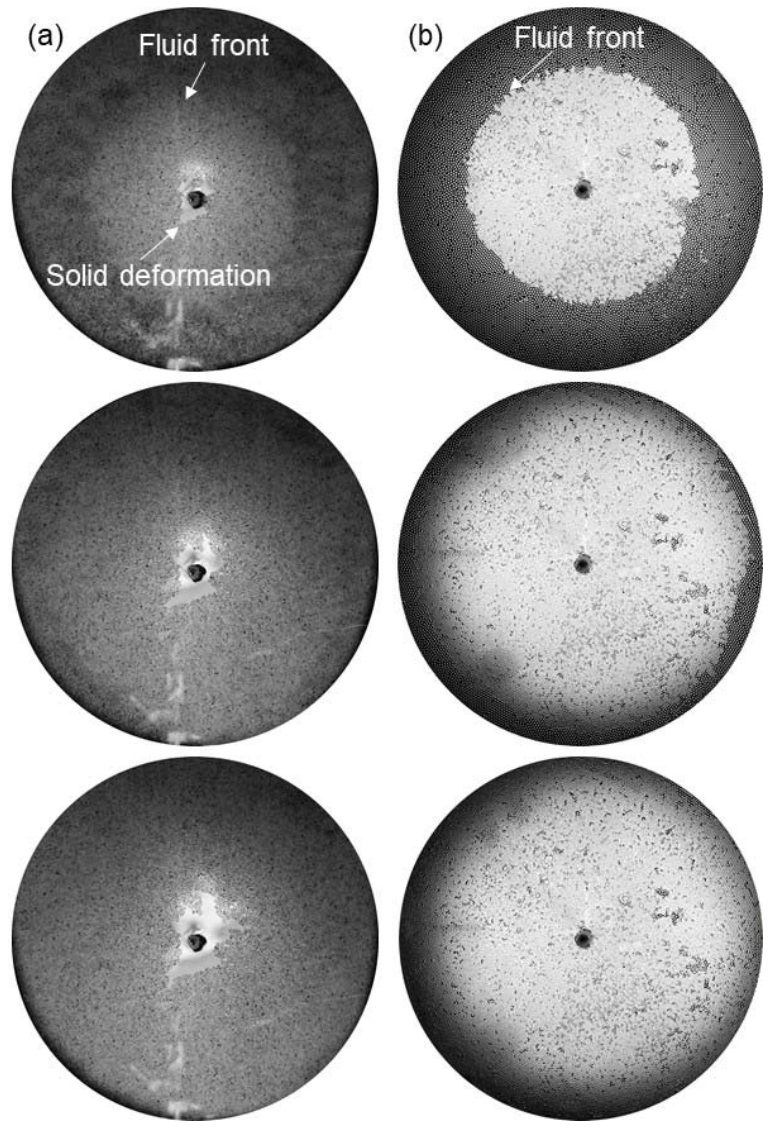

Fig. 2. Time-lapse images of fluid flow and the deformation of solid grains during the mineral oil injection into the watersaturated (a) hydrogel medium, and (b) glass bead medium. 
The injection of higher-viscous mineral oil into the water-saturated and deformable porous medium resulted in the fracture-like deformation of solid grains. Those fractures propagated with particle rearrangement as the mineral oil injection continued. Note that the fluid flow pattern was almost radially uniform, while the deformation of solid grains was severely localized. We also observed the deformation of solid grains was recovered after a couple of minutes when the injection was terminated.

On the other hand, the injection of mineral oil into the stiff porous medium (glass beads) did not induce any substantial deformation of solid grains or the structural rearrangement of them. Only minute mini fractures were observed near the injection point. And the injected fluid flows quite uniformly in the pore structure until percolation (e.g., white color in the most domain of porous medium - Fig. $2 \mathrm{~b}$ ). That comparison reveals the important effect of grain stiffness. That is, fluid flow in the soft porous medium involves two current phenomena, the fluid flow through the pores and deformation of solid grains, while the only fluid flow has predominantly occurred in the porous medium that consists of stiff glass beads.
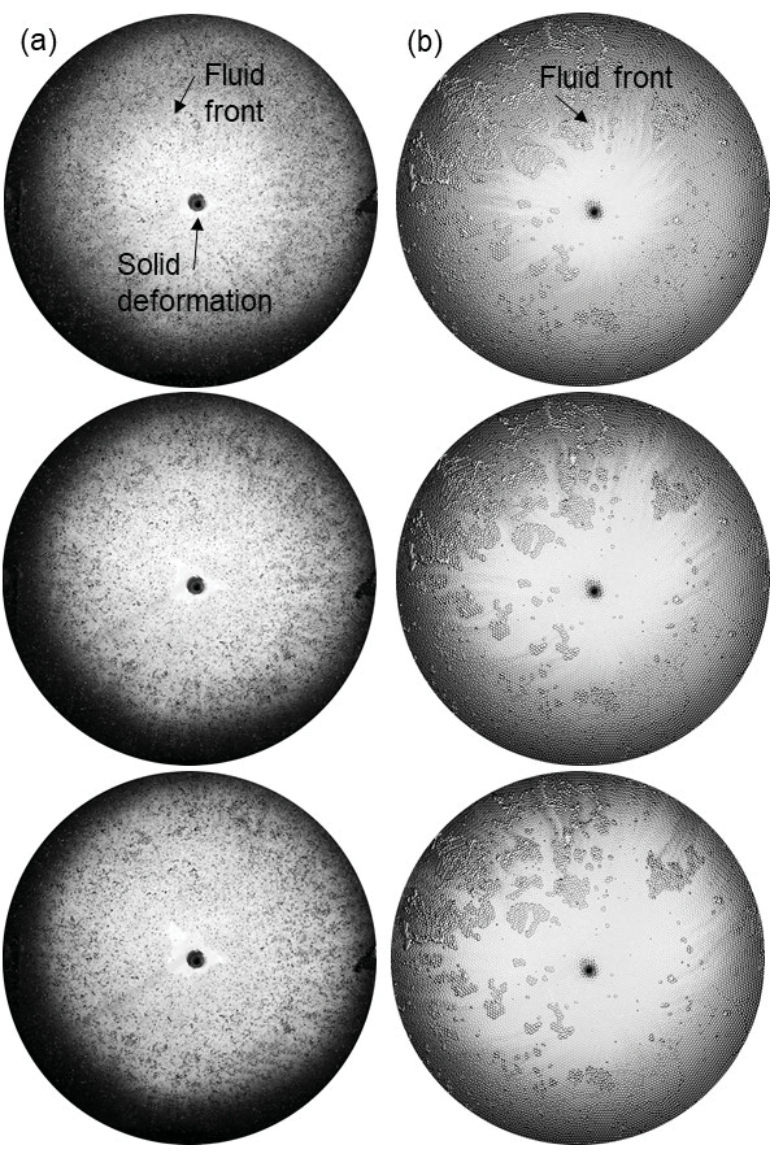

Fig. 3. Time-lapse images of fluid flow pattern and the deformation of solid grains during the same fluid injection (the water-glycerol mixture) into the fluid-saturated (a) hydrogel medium, and (b) glass bead medium.
We also investigated the results of single-phase fluid flow in both deformable and non-deformable porous media (Fig. 3a and 3b). The high viscosity of fluid resulted in the deformation of solid grains in the vicinity of the injection port, but its shape is rather radially uniform compared to the fracture-like shape of two-phase fluid flow (compare Fig. 2a and 3a). With that, we can conjecture that the presence of capillarity would be the main factor that leads to the localized development of solid deformation during the fluid flow, while the viscosity-driven deformation is developed more uniformly. Moreover, the size of the solid deformation is much smaller than that of two-phase fluid flow conditions under the same injection rate. On the other hand, the fluid flow pattern in the non-deformable porous medium is fairly identical - radially uniform flow without any substantial solid deformation.

\section{Numerical Investigation}

\subsection{Hydro-mechanically coupled pore network model (HM-PNM)}

We further investigate the two-phase fluid flows in deformable porous media using a numerical approach. The block-spring (BS) model and the pore network model (PNM) are coupled to simulate the concurrent two-phase flow and the medium deformation. The two-dimensional square lattice configuration is used to simplify the geometry [13].

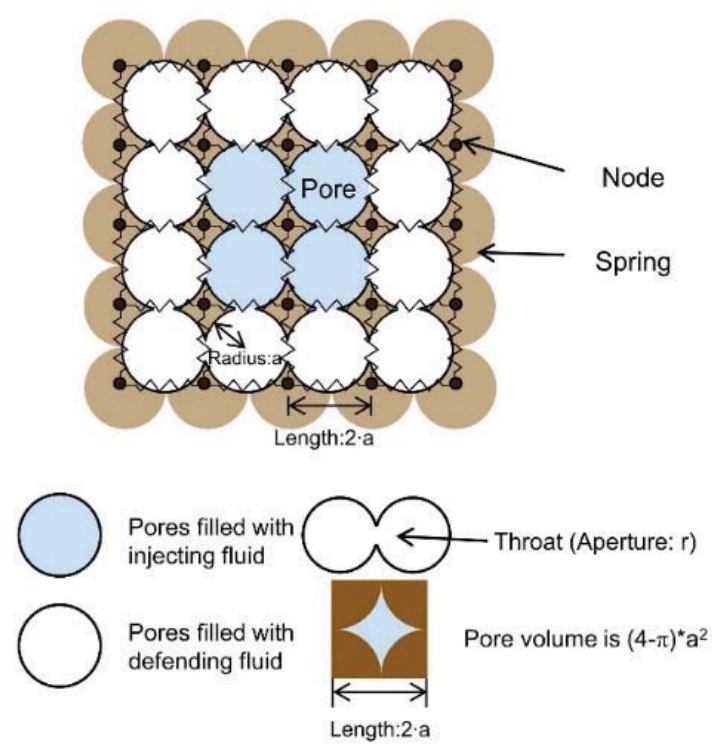

Fig. 4. A schematic of H-M coupled PNM (adopted from [16]).

The BS model is composed of nodes and springs. The nodes represent the particle position, while the springs represent the contact force between particles. We assume that the contact behavior follows the Hertzian contact model [14]. The particle radius is set equal to the grain radius, $a$, i.e., the distance between two nodes is $2 \cdot a$. Before fluid injection starts, all springs are under small isotropic stress that causes the initial spring contraction of 
$h_{0}$. The springs have non-linear spring stiffness $K$, derived from the Hertzian contact model (Eq.1).

$$
K=2 E^{*} \sqrt{a h / 2},
$$

where $E^{*}$ is the effective elastic modulus of solid particles, and $h$ is the spring contraction [14]. According to Eq.1, as the spring contracts, the larger force is required to contract the same amount of spring.

The pore space that fluids pass through is embodied using 2D PNM, which has a spherical pore area (Fig. 4). Each sphere-like pore is connected by a throat, which is a flow path from pore to pore. All pores are directly connected to the adjacent pores; so that it represents as circular shape with radius $a$, while their volume is (4$\pi) \cdot a^{2}$. The aperture of the throat $(r)$ determines the flow characteristics. We assume a Stokes flow in cylindrical tubes of radius $r$, where the local permeability of each throat is proportional to the square of throat aperture $\left(k=r^{2} / 8\right)$. The capillary pressure $\left(P_{c}\right)$ between two fluids follows the Young-Laplace equation $\left(P_{c}=2 \cdot \sigma / r\right)$, where $\sigma$ is the interfacial tension between two fluids. The effect of wettability is not considered to simplify the simulation. The throat aperture is generated with an uniform distribution from $(1-\lambda) \cdot \mathrm{a} / 2$ to $(1+\lambda) \cdot \mathrm{a} / 2$, where $\lambda$ is the disorder coefficient $\lambda \in(0,1)$.

At the beginning of the simulation, the central four pores are filled with an invading fluid, and the injection pressure is imposed on these four inlet pores. The pressure of the boundary pores is assumed to be zero. The pressure distribution is then calculated by assuming the steadystate and using the mass conservation equation $(\Sigma Q-$ pore $=0)$. The fluid displacement follows Darcy's law, $Q=(A \cdot k \cdot \Delta P) /\left(\mu_{e f f} 2 \cdot a\right)$, where $Q$ is the flow rate, $A$ is pore area, $\Delta P$ is the pressure difference between pores, and $\mu_{\text {eff }}$ is the effective viscosity. The effective viscosity is determined to be a weighted average value by using the volume fraction of each fluid between two pores. When the immiscible fluid is injected, the capillary pressure between two pores acts in the opposite direction to the fluid displacement. Therefore, invading fluid can flow only if the pressure difference between pores is larger than the capillary pressure $\left(\Delta P>P_{c}\right)$.

Before starting the fluid injection, the whole system is in the force equilibrium. The pore pressure change arises when the injection pressure is applied to the inlet pores. The change of pore pressure affects the adjacent nodes, where the contact forces change to maintain the force equilibrium at each node. The contact force in the BS model can be expressed by the product of spring stiffness and spring contraction, according to Hooke's law. The change of spring contraction $(\Delta h)$ is calculated, as follows:

$$
\Sigma\left(f_{p}(t+\Delta t)-f_{p}(t)\right)+\Sigma K \Delta h=0,
$$

where the forces exerted by pore pressure is $f_{p}$. The nodal position of each particle is then updated using the spring contraction values $(h)$ and the fixed boundary condition. The fluid-driven deformation affects the fluid conductance by changing the throat aperture $(\Delta r)$. The amount of aperture change can be derived from the Hertzian contact model as follows [14]:

$$
\Delta r=-\frac{\Delta h(1-\varepsilon)}{2 \sqrt{1+(1-\varepsilon)^{2}}},
$$

where $\varepsilon$ is the strain of each spring $(\varepsilon=h / l)$. Using these relations, the interplay of fluid flow and the mechanical deformation is embodied in HM-PNM.

\subsection{Numerical simulation results}

We model viscous fluid injection into the water-saturated porous medium under a constant flow rate, the similar condition with the experiments. Table 1 summarizes the input parameters used in the reference case. The twophase flow simulations are implemented with the moderately disordered pore-network with $\lambda=0.3$. The effects of medium stiffness, IFT between two fluids, and fluid viscosity are investigated by changing these parameters.

The HM-PNM simulation results are presented using different colors and lines hereafter. For example, the saturation of invading fluids at each pore is expressed with a variation of colors. When the pore saturation value is closer to 1 , it means the pore is filled with invading fluid. A percolated pore is expressed with yellow (see the color bar in Fig. 5). The intersection of lines represents the position of each node.

Table 1. Input parameters for HM-PNM

\begin{tabular}{|c|c|}
\hline Input parameter & Values \\
\hline \hline Size of pore-network & $50 \times 50$ \\
\hline Pore radius, $a$ & $1 \mathrm{~mm}$ \\
\hline $\begin{array}{c}\text { Effective elastic modulus of particles, } \\
E^{*}\end{array}$ & $50 \mathrm{kPa}$ \\
\hline Viscosity of invading fluid, $\mu_{\text {in }}$ & $10 \mathrm{mPa} \cdot \mathrm{s}$ \\
\hline Viscosity of defending fluid, $\mu_{\text {def }}$ & $1 \mathrm{mPa} \cdot \mathrm{s}$ \\
\hline flow rate, $q$ & $10^{-6} \mathrm{~m}^{2} / \mathrm{s}$ \\
\hline Disorder coefficient, $\lambda$ & 0.3 \\
\hline IFT between two fluids & $45 \mathrm{mN} / \mathrm{m}$ \\
\hline
\end{tabular}

\subsubsection{Effect of medium stiffness}

The influences of medium stiffness on fluid flow, hydromechanical deformation, and injection pressure are numerically examined first using the HM-PNM. The stiffness of grain and porosity determines the porous medium stiffness [15]. We compare two cases with the effective elastic modulus of particles $E^{*}=50 \mathrm{kPa}$ and with $E^{*}=5 \mathrm{MPa}$. All the other conditions, other than the grain stiffness, are kept the same (Table 1). In this condition, capillary number $(\mathrm{Ca})$ and viscosity ratio $(\mathrm{M})$ are $1.05 \times 10^{-7}$ and 10 , respectively. A significant deformation is developed in the soft medium $\left(E^{*}=50\right.$ $\mathrm{kPa}$ ), as shown in Fig. 5a. By contrast, little hydromechanical deformation is observed in the stiffer medium (Fig. 5b). This result demonstrates that the medium stiffness is one of the most important feature 
which determines the evolution of fluid-driven deformation. Meanwhile, the medium stiffness is found to have only a minimal effect on fluid flow patterns, showing very similar, radially invading patterns. These numerical simulation results are consistent with the experimental observations (Fig. 2), and this validates our HM-PNM.

As the mechanical deformation proceeds, the rate of pressure build-up decreases (Fig. 5c). The change of throat aperture $(\Delta r)$ accompanies the solid deformation associated with the spring contraction $(\Delta h)$ in the BS model (Eq.3). Thereby, the fluid-driven deformation enlarges the fluid path, which increases the fluid conductivity and lowers the capillary pressure. This phenomenon is called a pore opening effect. With continued injection, the inlet pressure in the softer medium becomes significantly lower than that in the stiff medium due to the accumulated pore opening effect (Fig. $5 \mathrm{c})$. This result reveals that the hydromechanical deformation lowers the pressure build-up.

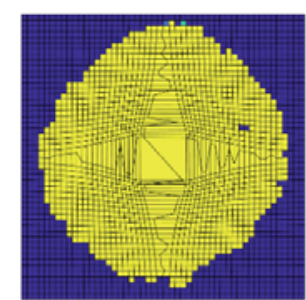

(a) $E^{*}=50 \mathrm{kPa}$

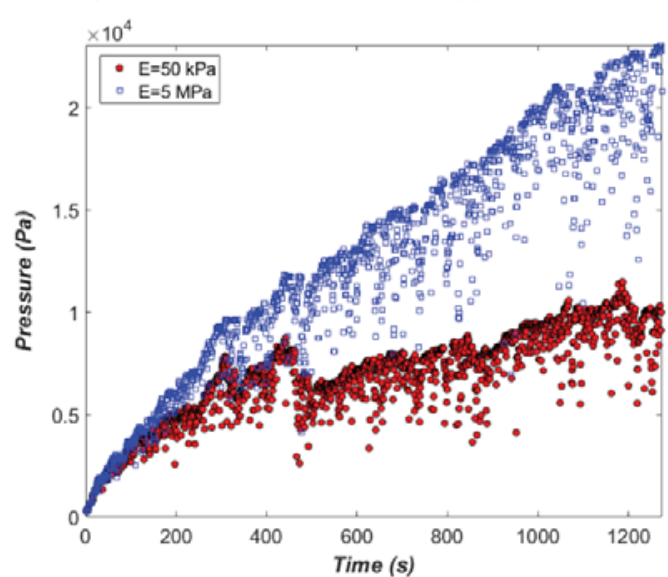

(c) Pressure response with time

Fig. 5. The effect of medium stiffness on the HM-PNM simulation results.

\subsubsection{Effect of interfacial tension (IFT)}

Reduction of IFT between two fluids can aid in enhancement of the recovery of oil and gas from a reservoir (i.e., enhanced oil recovery). We test the effect of reduced IFT on fluid-driven deformation. Herein, the IFT value is reduced from 45 to $10 \mathrm{mN} / \mathrm{m}$ while increasing the capillary number $(\mathrm{Ca}=\mu V / \sigma)$. As shown in Figs. $6 \mathrm{a}$ and $6 \mathrm{~b}$, the IFT appears to have little impact on the flow pattern. Our flow condition for the reference case lies in the transition between the stable displacement regime and the capillary fingering regime, i.e., $\log (\mathrm{M}) \approx 1$, $\log (\mathrm{Ca}) \approx-6.9$ [8-12]; the stable displacement pattern thus appears to be dominant even with the high IFT (45 $\mathrm{mN} / \mathrm{m}$ ). Accordingly, in the given situation, the increase in the capillary number with the low IFT value $(10 \mathrm{mN} / \mathrm{m})$ barely affects the flow pattern. Capillary number slightly increased from $1.05 \times 10^{-7}$ to $1.09 \times 10^{-7}$ by IFT reduction.

Instead, the IFT alteration contributes to reduce the capillary pressure at each throat, which lowers the injection pressure (Fig. 6c). The level of pore pressure buildup in the case with the low IFT value is much smaller, which lead to the smaller mechanical deformation, compared to the case with the high IFT value (Fig. 6a-b). This result implies that the IFT reduction not only increases the fluid mobility but also prevents the mechanical damage of hosting media.

One notable observation is the difference in the temporal evolution of injection pressure. When the IFT is lowered, the pressure response shows a significantly lower value from the beginning of fluid injection (Fig. $6 c$ ). On the other hand, when the medium stiffness is reduced, the inlet pressure is equivalent at the beginning but gradually decreases as hydromechanical deformation is developed (Fig. 5c).

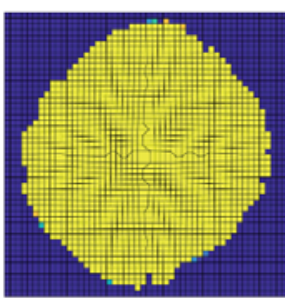

(a) IFT $10 \mathrm{mN} / \mathrm{m}$

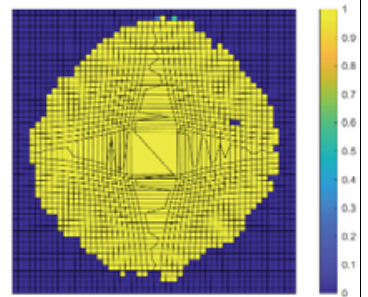

(b) IFT $45 \mathrm{mN} / \mathrm{m}$

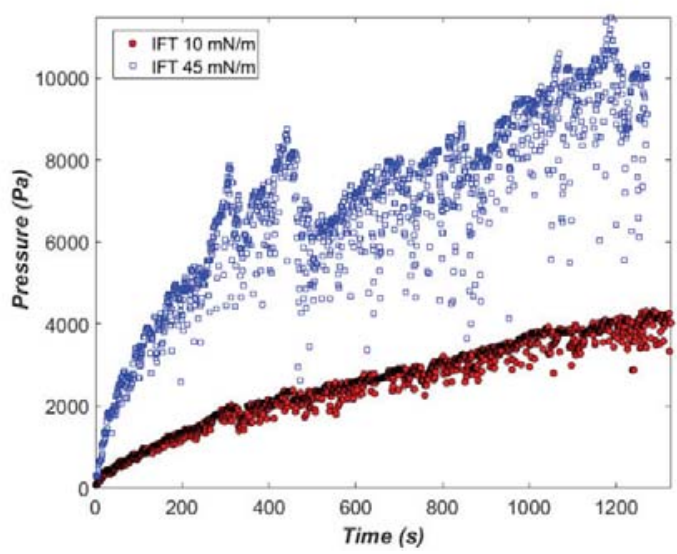

(c) Pressure evolution with time

Fig. 6. Effect of interfacial tension

\subsubsection{Effect of viscosity}

We numerically examine the effect of viscosity of the invading fluid by varying the viscosity from 0.01 to 10 $\mathrm{mPa} \cdot \mathrm{s}$. Figs. 7a-to-7d show the different fluid patterns associated with fluid viscosity (Figs. 7a-to-7d). When a low viscosity fluid is injected, the fluid flow exhibits a narrow preferential viscous fingering pattern. In addition, the medium deformations are minimal (Figs. 7a and 7b). As the viscosity of invading fluid increases, the fluid 
pattern moves from viscous fingering to stable displacement, and the more pronounced deformation occurs (Figs. 7a-to-7d). In particular, a significant transition in the flow pattern is observed when the viscosity of invading fluid becomes similar to that of defending fluid (Fig. 7e).

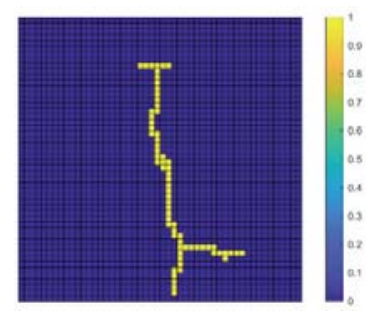

(a) $\mu_{\text {in }}=0.01 \mathrm{mPa} \cdot \mathrm{s}$

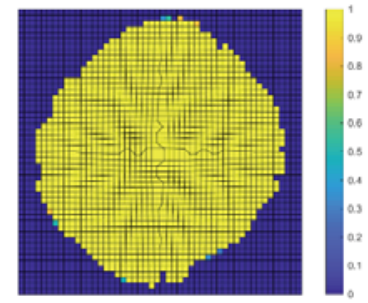

(c) $\mu_{\text {in }}=1 \mathrm{mPa} \cdot \mathrm{s}$

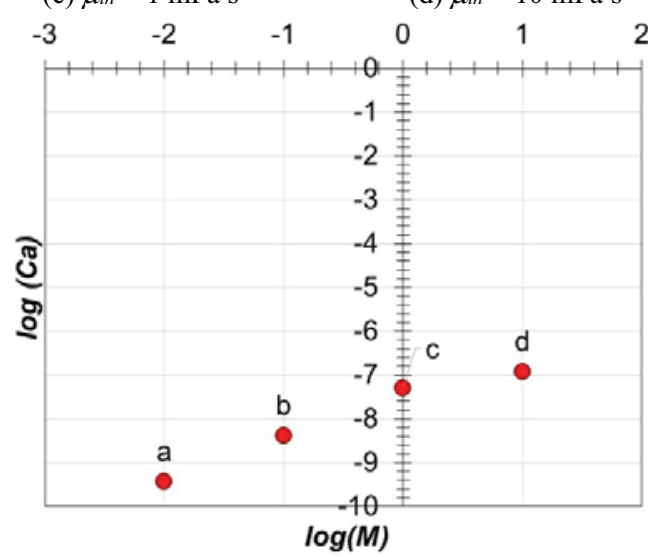

(e) Two-phase flow regimes associated with $\mathrm{Ca}$ and $\mathrm{M}$

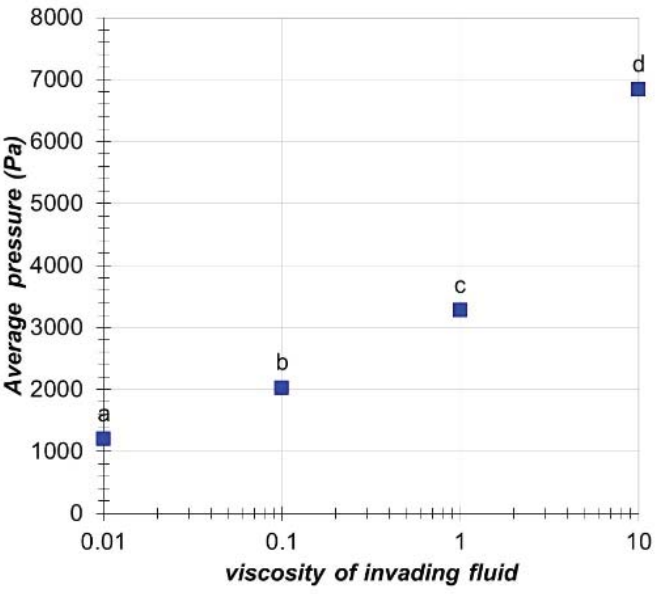

(f) Average pressure with invading fluid viscosity

Fig. 7. Effect of invading fluid viscosity

These flow pattern transitions are in a good agreement with the previous researches [8-12]. Although the previous studies were performed without considering the effect of host deformation, the flow patterns are still very similar to our simulation results. As the more viscous fluid is injected, the higher fluid saturation of invading fluid is achieved at percolation (Figs 7a-to-7d). The effective viscosity associated with the invading fluid affects the hydraulic conductance $\left((A \cdot k) /\left(\mu_{\text {eff }} 2 \cdot a\right)\right)$. More viscous invading fluid $(\mathrm{M}>1)$ results in the less hydraulic conductance, and it increases the injection pressure (Fig. 7f) and yields more deformation (Figs. 7a-to7-d).

\section{Conclusion}

In this study, we investigated the hydro-mechanically coupled behavior under the viscous fluid injection using the experimental and numerical methods at the pore scale. The patterns of fluid flow and deformation/displacement of solid particles were monitored during both single- and two-phase flows in the laboratory tests. The medium deformation occurred in the hydrogel pack, while a minimal movement was observed in the non-deformable glass pack. The HM-PNM simulation of two-phase flow with different mechanical stiffness values shows the results consistent with those experimental test results. Using HM-PNM, the effect of interfacial tension and viscosity of the invading fluid were examined further in detail. The IFT alteration and reduced capillary pressure lower the necessary injection pressure. The viscosity of invading fluid affects the capillary number and viscosity ratio, which determines the flow regime. The flow regimes are intimately related to the injection pressure, and so the particle deformation is also significantly affected by the viscosity of injection fluid.

This work was financially supported by Korea Minister of Ministry of Land, Infrastructure and Transport (MOLIT) as "Innovative Talent Education Program for Smart City", and by a grant (19CTAP-C151917-01) from Technology Advancement Research Program (TARP) funded by Ministry of Land, Infrastructure and Transport of Korean government. A. Zadeh and S. Kim are supported by Research Council: Interdisciplinary Grant at University of Nebraska-Lincoln.

\section{References}

1. D. Massonnet, T. Holzer, H. Vadon, "Land subsidence caused by the East Mesa geothermal field, California, observed using SAR interferometry", Geophys. Res. Lett., 24(8): p. 901-904 (1997)

2. J.E. Streit, R.R. Hillis, "Estimating fault stability and sustainable fluid pressures for underground storage of $\mathrm{CO} 2$ in porous rock", Energy, 29(9): p. 1445-1456 (2004)

3. P. Pan, Z. Wu, X. Feng,F. Yan, "Geomechanical modeling of $\mathrm{CO} 2$ geological storage: A review", Journal of Rock Mechanics and Geotechnical Engineering, 8(6) p. 936-947 (2016)

4. S.N. Pandey, A. Chaudhuri, S. Kelkar, "A coupled thermohydro-mechanical modeling of fracture aperture alteration and reservoir deformation during heat extraction from a geothermal reservoir", Geothermics., 65 p. 17-31 (2017)

5. A. Kharaghani, T. Metzger, E. Tsotsas, "A proposal for discrete modeling of mechanical effects during drying, 
combining pore networks with DEM", AICHE J., 57(4) p. 872-885 (2011)

6. R. Holtzman, M.L. Szulczewski, R. Juanes, "Capillary fracturing in granular media", Phys. Rev. Lett., 108(26): p. 264504 (2012)

7. C.W. MacMinn, E.R. Dufresne, J.S. Wettlaufer, "Fluiddriven deformation of a soft granular material", Phys.Rev. X., 5(1): p. 011020 (2015)

8. R. Lenormand, E. Touboul, C. Zarcone, "Numerical models and experiments on immiscible displacements in porous media", J. Fluid Mech., 189: p. 165-187 (1988)

9. X. Zheng, N. Mahabadi, T.S. Yun, J. Jang, "Effect of capillary and viscous force on $\mathrm{CO} 2$ saturation and invasion pattern in the microfluidic chip", J. Geophys. Res. Solid Earth., 122(3): p. 1634-1647 (2017)

10. C. Chang, T.J. Kneafsey, Q. Zhou, M. Oostrom,Y. Ju, "Scaling the impacts of pore-scale characteristics on unstable supercritical $\mathrm{CO}$-water drainage using a complete capillary number", Int. J. Greenh. Gas Con., 86: p. 11-21 (2019)

11. E. Aker, K. JØrgen MÅlØy, A. Hansen, G.G. Batrouni, “A two-dimensional network simulator for two-phase flow in porous media", Transport Porous Med., 32(2): p. 163-186 (1998)

12. G.M. Badillo, L.A. Segura,J.B. Laurindo, "Theoretical and experimental aspects of vacuum impregnation of porous media using transparent etched networks", Int. J. Multiphase Flow, 37(9): p. 1219-1226 (2011)

13. R. Holtzman, R. Juanes, "Crossover from fingering to fracturing in deformable disordered media", Phys. Rev. E., 82(4): p. 046305 (2010)

14. K.L. Johnson, Contact mechanics (Cambridge University Press, 1987)

15. L.F. Nielsen, "Strength and stiffness of porous materials", J. Am. Ceram. Soc., 73(9): p. 2684-2689 (1990)

16. M.K. Jeon, S. Kim, A. Hosseini Zadeh, T.H Kwon, "Study on Viscous Fluid Flow in Disordered-Deformable Porous Media Using Hydro-mechanically Coupled Pore-Network Modeling”, Transport Porous Med., 133: p. 207-227(2020) 